\title{
ON A THEOREM OF BEHREND
}

\author{
P. ERDŐS, A. SARKÖZY and E. SZEMERÉDI
}

(Received 15 September 1965)

\section{Dedicated to the memory of Felix Behrend}

A sequence of integers $0<a_{1}<a_{2}<\cdots$ no term of which divides any other will be called a primitive sequence. Throughout this paper $c_{1}, c_{2}, \cdots$ will denote suitable positive absolute constants. Behrend [1] proved that for every primitive sequence

$$
f_{A}(x)=\sum_{a_{i}<x} \frac{1}{a_{i}}<c_{1} \log x /(\log \log x)^{\frac{1}{2}} .
$$

Sivasankaranarayana Pillai observed that $(1)$ is in a sense best possible. He showed that there is a $c_{2}$ so that for every $x$ there is a primitive sequence $a_{1}<\cdots<a_{k} \leqq x$ for which

$$
f_{A}(x)>c_{2} \log x /(\log \log x)^{\frac{1}{2}}
$$

In the present paper we are going to prove the following

TheOREM 1. Let $A$ be an infinite primitive sequence. Then

$$
f_{A}(x)=o\left(\log x /(\log \log x)^{\frac{1}{2}}\right) .
$$

Our Theorem shows that though for a finite primitive sequence (1) is best possible, it can nevertheless be improved for infinite primitive sequences.

Before proving our Theorem we show that it is best possible. In fact we shall show that if $h(x) \rightarrow \infty$ arbitrarily slowly, then there exists a primitive sequence $A$ so that

$$
\limsup _{x \rightarrow \infty} f_{A}(x) h(x)\left(\log \log x^{\frac{1}{2}}\right) / \log x=\infty .
$$

We only outline the proof of (4) since the details can easily be filled in by the reader using the methods of [3]. Let $x_{1}<x_{2}<\cdots$ tend to infinity sufficiently fast. In the interval $\left(x_{\nu-1}, x_{\nu}\right)$ our sequence consists of the integers having exactly $\left[\log \log x_{\nu}\right]$ distinct prime factors greater than $x_{y-1}$ (and no prime factor $\leqq x_{p-1}$ ). A simple computation shows that if $x_{v} \rightarrow \infty$ sufficiently fast (depending on $h(x)$ ) then (4) holds. 
To prove Theorem 1 we assume that there is a primitive sequence $A=\left\{a_{1}<a_{2}<\cdots\right\}$ for which (3) does not hold and we will obtain a contradiction. First of all we observe that if a sequence $A$ exists for which (3) does not hold, we can assume that there is such a sequence all whose terms are squarefree. Put

$$
A=\bigcup_{k=1}^{\infty} A^{(k)}
$$

where the greatest square factor of the integers of $A^{(k)}$ is $k^{2}$. It easily follows from $\sum_{k=1}^{\infty} 1 / k^{2}<\infty$ and (1) that if $A$ does not satisfy (3) then for some fixed $k_{0}, A^{\left(k_{0}\right)}$ also does not satisfy (3). Put

$$
A^{\left(k_{0}\right)}=\left\{a_{1}^{\left(k_{0}\right)}<a_{2}^{\left(k_{0}\right)}<\cdots\right\} .
$$

Clearly $a_{i}^{\left(k_{0}\right)}=k_{0}^{2} b_{i}$ where $b_{i}$ is squarefree and $b_{1}<b_{2}<\cdots$ evidently does not satisfy (3).

Henceforth we assume that $A$ is a primitive sequence of squarefree numbers for which (3) does not hold. Then there clearly exists a sequence $x_{1}<x_{2}<\cdots$ tending to infinity sufficiently fast (this will be specified later) so that

$$
\sum_{x_{v-1}<a_{i}<x_{v}} \frac{1}{a_{i}}>c_{3} \log x_{v} /\left(\log \log x_{v}\right)^{\frac{1}{2}}
$$

We shall show that (5) leads to a contradiction and this will prove Theorem 1.

We need the following crucial

Lemma 1. Let $u<w \leqq y$, where $w$ is sufficiently large compared to $u$. Let (the a's are squarefree)

$$
u<a_{1}<\cdots<a_{k}<w, a_{i} \nmid a_{3}, \quad 1 \leqq i<j \leqq k
$$

and

$$
\sum_{i=1}^{k} \frac{1}{a_{i}}>c_{3} \log w /(\log \log w)^{\frac{1}{t}}
$$

Denote by $b_{1}<\cdots<b_{s} \leqq y$ the integers of the form

$$
a_{i} Q_{m}, \quad Q_{m} \leqq y / a_{i}, \quad 1 \leqq i \leqq k
$$

where all the prime factors of $Q_{m}$ are greater than $u$. Then

$$
\sum_{i=1}^{\infty} \frac{1}{b_{i}}>c_{4} \log y
$$

where $c_{4}$ depends only on $c_{\mathrm{a}}$.

Assume that Lemma 1 has already been proved then we prove Theorem 1 as follows: Let $\lambda c_{4}>2, y=x_{\lambda}$. For each $1 \leqq \nu \leqq \lambda$ we denote by $B^{(v)}$ the sequence of integers $b_{1}^{(\nu)}<\cdots<b_{v_{v}}^{(\nu)}$ of the form 


$$
a_{i} Q_{m}^{(\nu)}, x_{\nu-1}<a_{i}<x_{\nu}, \quad Q_{m}^{(\nu)}<x_{\nu} / a_{i}
$$

where all prime factors of $Q_{m}^{(\nu)}$ are greater than $x_{\nu-1}$. By Lemma 1 we have

$$
\sum_{i=1}^{s_{v}} \frac{1}{b_{i}^{(\nu)}}>c_{4} \log y \text {. }
$$

Now we show that

$$
B^{(\nu)} \cap B^{\left(\nu^{\prime}\right)}=\emptyset \quad \text { if } \quad \nu \neq v^{\prime} ;
$$

in other words $b_{i}^{(\nu)} \neq b_{i}^{\left(\nu^{\prime}\right)}$ if $\nu^{\prime}>v$. If (9) would be false we would have $a_{i} Q_{m}^{(\nu)}=a_{j} Q_{n}^{\left(\nu^{\prime}\right)}$ or $a_{i} / a_{j} Q_{n}^{\left(\nu^{\prime}\right)}$. But by our definitions $a_{i}<x_{\nu}, a_{3}>x_{\nu^{\prime}-1} \geqq x_{\nu}$, thus $a_{i}<a_{i}$ and hence $a_{i} \nmid a_{j}$. On the other hand all prime factors of $Q_{n}^{\left(\nu^{\prime}\right)}$ are greater than $x_{\nu^{\prime}-1} \geqq x_{\nu}$. Thus $\left(a_{i}, Q_{n}^{\left(\nu^{\prime}\right)}\right)=1$, hence $a_{i} / a_{j} Q_{n}^{\left(\nu^{\prime}\right)}$ implies $a_{i} / a_{i}$, an evident contradiction. Hence (9) is proved. Clearly $b_{i}^{(\nu)}<y$ for $v \leqq \lambda$. Thus by (8), (9) and $\lambda c_{4}>2$,

$$
2 \log y>\sum_{t<y} \frac{1}{t} \geqq \sum_{\nu=1}^{\lambda} \sum_{i=1}^{s_{\nu}} \frac{1}{b_{i}^{(\nu)}} \geqq \lambda c_{4} \log y>2 \log y
$$

an evident contradiction which proves Theorem 1 .

Thus to prove Theorem 1 we only have to prove Lemma 1 . We first assume $y=w$ and prove the Lemma in this special case. The general case will follow easily. Denote by $d_{1}(n)$ the number of divisors of $n$ amongst the $a_{i}, 1 \leqq i \leqq k, d_{2}(n)$ denotes the number of divisors of $n$ amongst the $b$ 's. The number of divisors $d(n)$ of the squarefree integer $n$ clearly equals $2^{\nu(n)}$ where $\nu(n)$ is the number of distinct prime factors of $n$. Clearly

$$
\sum_{n=1}^{\infty} d_{2}(n)=\dot{\sum}\left[\frac{w}{b_{i}}\right]<w \sum_{i=1}^{\dot{1}} \frac{1}{b_{i}} \text {. }
$$

Thus to prove Lemma 1 in the case $y=w$ it will suffice to show that

$$
\sum_{n=1}^{\infty} d_{2}(n)>c_{5} w \log w \text {. }
$$

Denote by $n_{1}<n_{2}<\cdots<n_{t}<w$ the sequence of integers satisfying

$$
\nu\left(n_{i}\right)>\log \log w \quad \text { and } \quad d_{1}\left(n_{i}\right)>c_{6} 2^{\nu\left(n_{i}\right)} / \nu\left(n_{i}\right)^{\frac{1}{2}}
$$

where $c_{B}$ is a sufficiently small constant which will be determined later. Clearly

$$
\sum_{i=1}^{t} d_{1}\left(n_{i}\right)=\sum_{n=1}^{\infty} d_{1}(n)-\Sigma^{\prime} d_{1}(n)-\Sigma^{\prime \prime} d_{1}(n)
$$

where in $\Sigma^{\prime}, n \leqq w$ and $v(n) \leqq \log \log w$, and in $\Sigma^{\prime \prime}$

$$
n \leqq w, v(n)>\log \log w, d_{1}(n) \leqq c_{6} 2^{\nu(n)} /(\nu(n))^{\frac{1}{2}}<c_{6} 2^{\nu(n)} /(\log \log w)^{\frac{1}{2}} .
$$


From (7) we evidently have

$$
\sum_{n=1}^{\infty} d_{1}(n) \geqq w \sum_{i=1}^{k} \frac{1}{a_{i}}-w>\frac{c_{3}}{2} w \log w /(\log \log w)^{\frac{1}{2}}
$$

Clearly

$$
\Sigma^{\prime} d_{1}(n)<w 2^{\log \log w}
$$

From (12) we have

$$
\begin{aligned}
\Sigma^{\prime \prime} d_{1}(n) & <c_{6} \sum_{n=1}^{\infty} \frac{2^{v(n)}}{(\log \log w)^{\frac{1}{2}}}<\frac{c_{8}}{(\log \log w)^{\frac{1}{2}}} \sum_{n=1}^{\infty} \frac{w}{n} \\
& <2 c_{8} w \log w /(\log \log w)^{\frac{1}{2} .}
\end{aligned}
$$

From (12), (14), (15) and (16) we have for $c_{0}<c_{3} / 10$

$$
\sum_{i=1}^{t} d_{1}\left(n_{i}\right)>\frac{c_{3}}{4} w \log w /(\log \log w)^{\frac{1}{2}}
$$

Thus to prove (10) we only have to show that for $1 \leqq i \leqq t$

$$
d_{2}\left(n_{i}\right)>c_{7} d_{1}\left(n_{i}\right)\left(\nu\left(n_{i}\right)\right)^{\frac{1}{2}}>c_{7} d_{1}\left(n_{i}\right)(\log \log w)^{\frac{1}{2}} .
$$

The last inequality of (18) follows from (11), (17) and (18) clearly imply (10).

To prove (18) let

$$
p_{1}<\cdots<p_{r_{1}} \leqq u<q_{1}<\cdots<q_{r_{2}} \leqq w
$$

be the prime factors of $n_{i}$. Clearly $r_{1}<u$, further by (11)

$$
r_{2}>\log \log w-u>\frac{1}{2} \log \log w>r_{1}
$$

if $w$ is sufficiently large (e.g. $w>\exp \exp 2 u$ ). Let $a_{1}, \cdots, a_{l}$ be the divisors of $n_{i}$ amongst the $a^{\prime}$ s. By (11)

$$
l>c_{8} 2^{v\left(n_{i}\right)} /\left(v\left(n_{i}\right)\right)^{\frac{1}{2}} .
$$

To obtain a lower bound for the number of $b$ 's dividing $n_{i}$, we multiply each $a / n_{i}$ by all the products of the $q$ 's which do not divide $a$. To show (18) we prove the following combinatorial

Lemma 2. Let $S=S_{1} \cup S_{2}, S_{1} \cap S_{2}=\emptyset$. The elements of $S_{1}$ are $e_{1}, \cdots, e_{k}$, the elements of $S_{2}$ are $f_{1}, \cdots, f_{l}$. Assume $l \geqq k$. Let $A_{i} \subset S, 1 \leqq i \leqq r$,

$$
r>c_{9} 2^{k+\bar{l}} /(k+l) \underline{\underline{z}}
$$

be subsets of $S$ no one of which contains any other. Denote by $B_{1}, \cdots, B_{i}$ all the (distinct) subsets of $S$ of the form 


$$
A_{i} \cup R, \quad 1 \leqq i \leqq r, \quad R \subset S_{2}
$$

where in (20) $R$ runs through all the $2^{l}$ subsets of $S_{2}$. Then

$$
t>c_{10} 2^{k+l} \text {. }
$$

(18) immediately follows from Lemma 2 (to see this it suffices to identify the $p$ 's with the $e$ 's and the $q$ 's with the $f^{\prime}$ 's (19) is satisfied because of (11).) Thus to complete the proof of Theorem 1 it suffices to prove Lemma 2.

Before proving Lemma 2 we first need

LEMMA 3. Let

$$
D_{i} \subset S_{2}, \quad 1 \leqq i \leqq j, \quad j>c_{1} 2^{2} / l^{\frac{1}{2}}
$$

be subsets of the set $S_{2}$ having $l$ elements where no $D$ contains any other. Let $E_{1}, \cdots, E$, be the set of all subsets of $S_{2}$ which contain at least one $D$. We have

$$
S>c_{14} 2^{l} \text {. }
$$

Denote by $\alpha_{r}\left(\begin{array}{l}n \\ r\end{array}\right)$ the number of those $D_{i}$ for which $\left|D_{i}\right|=r$ and by $\beta_{r}\left(\begin{array}{c}n \\ r\end{array}\right)$ the number of the $E$, satisfying $|E|=r(|A|$ is the number of elements of $A$ ). We first show

$$
\beta_{r} \geqq \sum_{j \leqq r} \alpha_{j},
$$

Lemma 3 can be deduced from (21) by a simple computation which we leave to the reader.

To prove (21) it clearly will be sufficient to show that

$$
\beta_{r} \geqq \beta_{r-1}+\alpha_{r} .
$$

Consider all the $E^{\prime}$ 's with $|E|=r-1$. Their number is $\beta_{r-1}\left(\begin{array}{l}n \\ r-1\end{array}\right)$. Consider now all sets of $r$ elements which contain one of these $E$ 's. Their number is $\beta_{r-1}\left({ }_{r-1}^{n}\right)(n-r+1)$ and the same set occurs at most $r$ times. Therefore the number of these sets is at least

$$
\beta_{r-1}\left(\begin{array}{l}
n \\
r-1
\end{array}\right) \frac{n-r+1}{r}=\beta_{r-1}\left(\begin{array}{c}
n \\
r
\end{array}\right) \text {. }
$$

These $\beta_{r-1}\left(\begin{array}{l}n \\ r\end{array}\right)$ sets are all $E$ 's satisfying $|E|=r$ and by assumption none of them are $D^{\prime}$ s having $r$ elements. Hence (21) is evident, and thus Lemma 3 is proved.

We conjectured and Kleitman proved the following stronger result: In a set $S$ of $n$ elements let there be given $\left(\begin{array}{l}n \\ r\end{array}\right)$ subsets of $S$

$$
D_{1}, \cdots, D_{\left(\begin{array}{l}
n \\
r
\end{array}\right)}, \quad D_{i} \notin D_{j}, \quad 1 \leqq i<j \leqq\left(\begin{array}{c}
n \\
r
\end{array}\right) .
$$


Denote by $E_{1}, \cdots, E_{\mathrm{a}}$ those subsets of $S$ which contain at least one of the $D$ 's. Then

$$
s \geqq \sum_{i=0}^{r}\left(\begin{array}{c}
n \\
i
\end{array}\right) .
$$

Now we prove Lemma 2. Put

$$
A_{i}=\left(A_{i} \cap S_{1}\right) \cup\left(A_{i} \cap S_{2}\right), \quad 1 \leqq i \leqq r .
$$

We split the class of all $A^{\prime}$ 's into $2^{k}$ classes $C_{1}, \cdots, C_{2^{k}}$ where two $A$ 's belong to the same class if they have the same intersection with $S_{1}$. Let $A_{i_{1}}$ and $A_{i_{2}}$ belong to the same class then $A_{i_{1}} \cap S_{2}$ clearly does not contain $A_{i_{2}} \cap S_{2}$. Hence by the theorem of Sperner [5] each class contains at most

$$
\left(\begin{array}{c}
l \\
{\left[\frac{1}{2} l\right]}
\end{array}\right)<c_{11} \frac{2^{l}}{l \frac{1}{2}}
$$

$A$ 's. From (22), (19) and $l \geqq k$ we obtain by a simple computation that there are at least $c_{12} 2^{k}$ classes which contain more than $c_{13} 2^{l} / l^{l} A^{\prime}$ 's. Denote these classes by $C_{i_{k}}, 1 \leqq k \leqq r, r>c_{12} 2^{k}$. By Lemma 3 the number of $B$ 's for which $B \subset S_{1} \cup S_{2}$ and $B \cap S_{2}=A \cap S_{2}$ where $A$ is in $C_{i_{k}}$ $(1 \leqq k \leqq r)$ is greater than $c_{14} 2^{2}$. Thus the number of $B$ 's is clearly greater than

$$
c_{12} c_{14} 2^{k+l}>c_{10} 2^{k+l}
$$

which proves Lemma 2 and therefore Lemma 1 in the case $y=w$.

To prove Lemma 1 in the general case denote by $1=t_{1}<t_{2}<\cdots$ the integers all whose prime factors are greater than $w$. We evidently have

$$
\sum_{b_{i}<y} \frac{1}{b_{i}} \leqq \sum_{b_{i}<w} \frac{1}{b_{i}} \sum_{t_{i}<v / w} \frac{1}{t_{i}} .
$$

We already proved Lemma 1 if $y=w$, hence

$$
\sum_{b_{i}<w} \frac{1}{b_{i}}>c_{15} \log w
$$

Further we obtain by a simple computation from a result of de Bruijn [2] that

$$
\sum_{t_{i}<y / w} \frac{1}{t_{i}}>c_{16} \log y / \log w .
$$

Lemma 1 clearly follows from (23), (24) and (25). Thus the proof of Theorem 1 is complete.

It is easy to see that Lemma 2 remains true for $l>c_{18} k$ but fails for $l=o(k)$.

We now state the following sharpening of Theorem 1: 
THEOREM 2. Let $A$ be a primitive sequence, $x_{1}, x_{2}, \cdots$ be any sequence satisfying

$$
\log \log x_{\nu+1}>\left(1+c_{17}\right) \log \log x_{\nu}
$$

where $c_{17}$ is an arbitrary constant. Put

Then

$$
\varepsilon_{\nu}=\frac{\left(\log \log x_{\nu}\right)^{\frac{1}{2}}}{\log x_{\nu}} f_{A}\left(x_{\nu}\right) .
$$

$$
\sum_{\nu=1}^{\infty} \varepsilon_{\nu}<c_{18}
$$

where $c_{18}$ depends only on $c_{17}$.

We do not give the proof of Theorem 2 since it is very similar to that of Theorem 1 and further (26) can probably be very much improved; perhaps Theorem 2 remains true if (26) is replaced by

$$
\log \log x_{\nu+1}>\log \log x_{\nu}+c_{19}\left(\log \log x_{\nu}\right)^{\frac{1}{2}}
$$

Theorem 1 gives the best upper bound for the growth of $f_{A}(x)$ for an infinite primitive sequence. Nevertheless further questions can be asked. A well known theorem [4] states that there is an absolute constant $c_{20}$ so that for every primitive sequence,

$$
\sum_{k} \frac{1}{a_{k} \log a_{k}}<c_{20}
$$

From (27) we obtain by partial summation

Now we prove

$$
\sum_{n} t_{A}\left(2^{2^{n}}\right) / 2^{n}<c_{21}
$$

THEOREM 3. Let $g(x)$ be an increasing function for which

$$
\sum_{n} g\left(2^{2^{n}}\right) / 2^{n}=\infty
$$

Then

$$
\lim \inf f_{A}(x) / g(x)=0 .
$$

On the other hand if $g_{1}(x)=\log x / \log \log x h(x)$ where $h(x)$ is increasing and $g_{1}(x)$ is also increasing and

$$
\sum_{n} g_{1}\left(2^{2^{n}}\right) / 2^{n}
$$

converges, then there is a primitive sequence for which

$$
\lim f_{A}(x) / g(x)=\infty \text {. }
$$


The first part of Theorem 3 follows immediately from (28), hence we only have to prove (29). We will leave some of the details to the reader. Let $p_{1}<p_{2}<\cdots$ be a sequence of primes for which $\sum 1 / p_{k}<\infty$ and $p_{k}=(1+o(1)) k \log k u(k)$ where $u(k)=o(h(k))$. By (29) such a choice is possible. Our primitive sequence consists of the integers of the form

$$
p_{k} t, \quad 1 \leqq k<\infty, \quad v(t)=k^{2}, \quad p_{i} \nmid t, \quad 1 \leqq i \leqq k .
$$

It is not difficult to show by using the methods of [3] that the number of $a_{i}$ not exceeding $x$ is greater than

$$
c_{22} x / u(x) \log \log x \text {. }
$$

In other words for all sufficiently large $n, a_{n}<c_{23} n u(n) \log \log n$ or

$$
f_{A}(x)>c_{24} \log x / u(x) \log \log x .
$$

In other words (30) holds. The monotonicity conditions on $g(x)$ could no doubt be relaxed, but we do not investigate this question.

\section{References}

[1] F. Behrend, 'On sequences of numbers not divisible one by another', J. London Math. Soc. 10 (1935), $42-45$.

[2] N. G. de Bruijn, 'On the number of uncancelled elements in the sieve of Eratosthenes', Indagationes Math. 12 (1950) 247-256.

[3] P. Erdos, 'On the integers having exactly $k$ prime factors', Annals of Math. 49 (1948), 53-66.

[4] P. Erdös, 'Note on sequences of integers no one of which is divisible by any other', $J$. London Math. Soc. 10 (1935), 126-128.

[5] E. Sperner, 'Ein Satz über Untermengen einer endlichen Menge', Math. Zeitschrift 27 $(1928), 644-548$. 